EXPANSIÓN VERTICAL DE LA CIUDAD: DENSIDAD URBANA, ¿DENSIDAD HUMANA? Milton Montejano Castillo*

\author{
VERTICAL EXPANSION OF THE CITY: URBAN \\ DENSITY OR HUMAN DENSITY?
}

RESUMEN: El futuro de las ciudades plantea su densificación y verticalización. Un incremento de la población en menos superficie disminuye desplazamientos, el consumo de suelo y el gasto de energía, aunque a costa de la interacción social y la salud mental. En el caso de la Ciudad de México, favorecer la verticalidad exige una cuidadosa adecuación al contexto local, natural e histórico.

PALABRAS ClaVE: Densificación, forma urbana, verticalización.
ABSTRACT: The future of cities gives rise to the idea of densification and verticalization. An increase of population in a smaller area reduces displacements, consumption of land and energy, although social interaction and mental health are subjects of discussion. In the case of Mexico City, favoring verticality would require a careful adaptation to the local, natural and historical context.

KEYWORDS: urban form, densification, verticalization.

* Escuela Superior de Ingeniería y Arquitectura Tecamachalco, Instituto Politécnico Nacional. 


\section{EXPANSIÓN VERTICAL DE LA CIUDAD: \\ DENSIDAD URBANA, ¿DENSIDAD HUMANA?}

\section{Introducción}

Mientras que ahora la ciudad compacta se promueve internacionalmente como el paradigma de una mejor calidad de vida y mayor cohesión de sus habitantes, también puede dar lugar a contradicciones entre la sociedad, el medio ambiente, la economía de una ciudad y su grado de compactación, expresado en su verticalidad. Así, cabe trazar un vínculo entre algunos aspectos de la forma urbana, la estética y el sentido de vida.

\section{La naturaleza económica de la edificación en altura}

Pareciera que las ciudades saturadas por rascacielos han sido el producto de una cuidadosa planificación con límites precisos y una intención común para el conglomerado de edificios verticales. Lo que a simple vista parece ser el crecimiento vertical ordenado de la ciudad, en gran parte obedece a razonamientos económicos individuales que dan por resultado la verticalidad o un uso más intenso del suelo.

Edwin Mills ${ }^{1}$ estableció la relación entre la superficie de suelo urbano y la intensidad de su uso en su

${ }^{1}$ Edwin S. Mills, "An aggregative model of resource allocation in a metropolitan area", The American Economic Review 57/2 (1967), pp. 197-210. 
modelo de "sustitución intraurbana de factores". Mills sostenía que la naturaleza e intensidad de uso del suelo varía de una ciudad a otra y de una parte de la ciudad a otra, como resultado de una sustitución de factores (tierra por capital), que el autor consideraba como la característica más radical de la estructura urbana.

En otras palabras, y para citar a Richardson, ${ }^{2}$ si se considera la ciudad como un lugar de producción, tres insumos o factores entran en juego: suelo, capital y trabajo. Para estos factores, se consideran como supuestos que el suelo, como soporte de las actividades urbanas y con localizaciones diferentes, tiene un valor que varía grandemente de un lado a otro de la ciudad, pero el capital y el costo de trabajo (salarios) se toman como constantes en el espacio intraurbano. Si pensamos en una ciudad con un solo centro, el gradiente de valor del suelo disminuirá a medida que nos acerquemos a la periferia.

Si se considera de manera muy simple esta función de producción y se adoptan los supuestos anteriores, en la periferia de la ciudad podrían adquirirse grandes superficies a un precio muy bajo, con una densidad de construcción igualmente muy baja. Conforme nos acerquemos a un centro de actividad económica, los precios del suelo en proporción a la

${ }^{2}$ Harry W. Richardson, Economía regional y urbana, 1986, Madrid, Alianza, p. 211. superficie del terreno serán más altos. En el momento en que se decide comprar o rentar este terreno, la sustitución de factores capital por suelo comienza a tener efecto. Se decidirá, por ejemplo, que las oficinas centrales se ubiquen en terrenos muy accesibles, que por su escasez y el deseo de sus propietarios por maximizar sus ganancias se organizarán en superficies de suelo pequeñas con un elevado costo por metro cuadrado. El número de trabajadores que requieren estas oficinas obligará nuevamente a las empresas a invertir una gran cantidad de capital para acomodar verticalmente a esta fuerza de trabajo y la relación entre trabajo y suelo será muy alta.

En la realidad entran en juego más factores que pueden repercutir en la altura de los edificios. La intensidad de uso también puede ser resultado de factores más subjetivos, como el afán de prestigio, simbolizado por edificios altos, aunque en el caso contrario, el fenómeno de encontrar edificios contiguos con otra altura (como las construcciones de uno o dos niveles en una zona de rascacielos) puede ser resultado de la falta de mecanismos de mercado que presionen a favor de una utilización más intensiva, como cuando los impuestos a la propiedad no reconocen el valor derivado del potencial de desarrollo. También puede darse el caso de una baja ocupación en áreas que originalmente se prestaban para la explotación intensiva, 
pero en las que fueron congeladas y canceladas las opciones de desarrollo e intensificación como consecuencia de la normatividad urbana. ${ }^{3}$

\section{La edificación en altura como objeto de estudio y discusión}

Actualmente, y partir de la cada vez mayor escasez de suelo y presión de crecimiento vertical en las ciudades, las edificaciones en altura se han convertido en un recurso de crecimiento en las ciudades con más presiones demográficas, y con ello, la verticalidad constructiva también se ha convertido en un elemento de discusión académica y un campo de investigación con al menos tres grandes ámbitos de análisis.

El primer grupo de estudios de la edificación en altura se relaciona con los aspectos urbanos y funcionales. Estos estudios se enfocan cuantitativamente en temas ambientales como la magnitud de las sombras que proyectan los edificios altos, el incremento de la velocidad del viento entre edificios de gran altura (efecto de túnel) y sus consecuencias en la dispersión de contaminantes, ${ }^{4}$ el consumo

${ }^{3}$ Irving Hoch, "La ciudad tridimensional: el espacio urbano cerrado", en Harvey S. Perloff (comp.), La calidad del medio ambiente urbano, 1973, Barcelona, Oikos-Tau Ediciones.

${ }^{4}$ Jian Hang, Yuguo Lia, Mats Sandberg, Riccardo Buccolieri y Silvana Di Sabatino, "The influence de suelo y los patrones de urbanización ${ }^{5} \mathrm{o}$ la presencia de edificios altos como factores que incrementan la demanda de transporte e infraestructura.

En el segundo grupo de análisis se encuentran los estudios sobre los aspectos de habitabilidad y calidad de vida de la producción de edificios en altura y sus usuarios. Gifford ${ }^{6}$ enumera los principales aspectos que se han investigado: satisfacción residencial y preferencias por vivir en edificios altos, angustia y salud mental, problemas de comportamiento, delincuencia y miedo a la delincuencia, comportamiento social, relaciones sociales y la vida infantil en edificios altos. Gifford concluye, aunque con mucha cautela, que en la mayoría de los aspectos mencionados vivir en edificios altos tiene más consecuencias negativas que positivas.

Finalmente, cabe reunir un tercer grupo de estudios de naturaleza más subjetiva, relacionados con aspectos de significado y percepción, pues así como ciertos edificios altos se han convertido incluso en iconos de ciudades, también son susceptibles de

of building height variability on pollutant dispersion and pedestrian ventilation in idealized high-rise urban areas", Building and Environment 56 (2012), pp. 346-360.

${ }^{5}$ Luca Salvati, Marco Zitti, y Adele Sateriano, "Changes in city vertical profile as an indicator of sprawl: Evidence from a Mediterranean urban region", Habitat International 38 (2013), pp. 119-125.

${ }^{6}$ Robert Gifford, "The consequences of living in high-rise buildings", Architectural Science Review 50/1(2007), pp. 2-17. 
ser percibidos como conjunto y como paisajes, según el concepto de "silueta urbana". En estos estudios se han explorado básicamente dos temas: la catalogación de vistas tanto para el habitante como para el visitante y el significado del horizonte urbano para la población. Una de las referencias básicas es el trabajo de Wayne Attoe, ${ }^{7}$ quien propone varios criterios para analizar la estética de la silueta urbana: el ritmo, la armonía, la proporción con el cielo, la forma, la secuencia y la yuxtaposición. Algunos autores consideran la complejidad de las siluetas como un factor constante en la preferencia de unas vistas sobre otras, pues - concluyen — cuanto más complejo es el tejido tridimensional que se aprecia a la vista, más atractivo resulta para los habitantes. Por último, se han propuesto criterios tomados de la teoría de la Gestalt como un método para interpretar la silueta urbana y sus posibles aciertos.

A pesar de su larga e ineludible presencia, la construcción de edificios altos (para usos habitacionales o no) aún sigue siendo objeto de discusión, como explican Ali y Al-Kodmany, ${ }^{8}$ al señalar los argumentos a favor y

${ }^{7}$ Skylines: Understanding and molding urban silhouettes, 1981, Nueva York, John Wiley $\&$ Sons Inc.

${ }^{8}$ Mir M. Ali y Kheir Al-Kodmany, "Tall buildings and urban habitat of the 21 st century: A global perspective", Buildings 2/4 (2012), pp. 384-423. en contra de estas edificaciones. Quizá uno de los aspectos de mayor controversia es que, mientras que para una visión funcional una forma urbana compacta que favorezca la densificación puede ser un medio para una mayor sostenibilidad, por otro lado, vivir en altura no condensa una manera de fomentar la interacción social y la cohesión. Esta discusión se ha desenvuelto en muchas parte del mundo, aunque en la Ciudad de México los aspectos relacionados con la verticalidad han sido escasamente explorados.

\section{Ciudad de México: una verticalidad en líneas}

En principio, dada la existencia de un centro histórico de baja densidad, en la Ciudad de México el uso intensivo del suelo o la verticalidad no se han dado tanto en polígonos, sino sobre todo a lo largo de ejes urbanos. La persistencia histórica de las vías de comunicación lineal en la estructura de ciudad latinoamericana ha sido mencionada por Terrazas; ${ }^{9}$ para el caso concreto de la Ciudad de México, explica que la concentración de actividades urbanas localizadas a lo largo de ejes y generalmente sobre

\footnotetext{
${ }^{9}$ Óscar Terrazas Revilla, "De la ciudad central a la ciudad interior", en Óscar Terrazas y Eduardo Preciat (coords.), Estructura territorial de la Ciudad de México, 1988, México, Plaza y Valdés/DDF, p. 86.
} 
las vías de comunicación ha acompañado su crecimiento y el de otras ciudades mexicanas prácticamente desde el periodo colonial. Terrazas señala igualmente que a pesar del crecimiento de la ciudad, no pudo mantenerse en funcionamiento el camino real, pero en el desarrollo histórico de la estructura de la metrópoli el fenómeno ha permanecido. El autor reafirma esta idea refiriéndose a las principales vías de comunicación y rutas de transporte en el interior de la ciudad como la representación de un patrón de ejes o caminos que corresponden a un concepto de centralidad replanteado, que no necesariamente coincide con el centro tradicional. ${ }^{10}$

De manera análoga, al referirse a la estructuración de la Ciudad de México, Pradilla describe cómo la antigua centralidad se organizó sobre sus ejes más importantes: "las subcentralidades se alargaron sobre los ejes de flujos materiales de personas y vehículos, hasta perder su forma concéntrica y articular a varios de ellos, entretejiéndose en una red". ${ }^{11}$ Sobre su composición, el autor dice que

${ }^{10}$ Terrazas Revilla (coord.), La ciudad que hoy es centro, 2010, México, UAM-A / CONACYT, p. 9.

${ }^{11}$ Emilio Pradilla Cobos, "La economía y las formas urbanas en América Latina”, en Blanca Rebeca Ramírez Velázquez y Emilio Pradilla Cobos (comps.), Teorías sobre la ciudad en América Latina, tomo I, 2013, México, Universidad Autónoma Metropolitana, pp. 226-227. estos corredores están compuestos por centros y plazas comerciales, pequeños y medianos comercios, oficinas bancarias y otras actividades financieras, servicios privados y públicos orientados a las actividades económicas, servicios de reparación, hoteles, restaurantes, lugares de entretenimiento y oficinas de gestión, entre otros usos.

Además, esta lógica es también la que priva en la planeación urbana, pues en la Ciudad de México el corredor urbano, en combinación con centros y subcentros urbanos, apoyados por el transporte y la red vial, ya se proponían en los Planes de Desarrollo Urbano de la década de 1980 como los componentes de una estructura urbana que debía constituir el "armazón físico" del Distrito Federal y que debía organizar el desarrollo urbano. En este plan se consideraba que la forma lineal de los corredores concedería una gran cobertura de toda la zona urbanizada para que se constituyeran franjas que alojaran las altas densidades de población y las mayores concentraciones de establecimientos de servicios y fuentes de trabajo, incluyendo industrias no contaminantes. ${ }^{12}$

A más de tres décadas, el concepto de corredor urbano sigue aparecien-

${ }^{12}$ Departamento del Distrito Federal, Secretaría de Obras y Servicios, Dirección General de Planificación, Plan General de Desarrollo Urbano del Distrito Federal, 1983, México, DDF. 
do en los planes de desarrollo urbano como elemento de una estructura policéntrica ${ }^{13}$ junto con ciertos nodos. Según la delegación, debe hacerse una distinción entre corredores de baja intensidad, corredores de carácter regional, de comercio y servicios.

Tanto las preexistencias históricas como las intenciones de planeación han moldeado estos elementos urbanos, pues en estudios de Montejano Castillo se observa que la verticalidad es, en efecto, uno de los rasgos distintivos de estos corredores urbanos, y que es el más característico, después del uso de suelo de umbral metropolitano y regional (oficinas, hoteles, centros comerciales), el uso habitacional multifamiliar mezclado con otros usos.

Una revisión bibliográfica más extensa sobre el tema en México revela que tanto la caracterización como la conformación histórica de dichos corredores ha sido el tema principal de las investigaciones, junto con aspectos de transporte y movilidad. En cambio, casi no se ha explorado la confrontación de la morfología de estos corredores con aspectos de habitabilidad, satisfacción con la vivienda, promoción de la interacción social $\mathrm{u}$ otros efectos de tipo cultural y psicológico.

${ }^{13}$ Gobierno del Distrito Federal, Programa General de Desarrollo del Distrito Federal 2013-2018. Gaceta Oficial del Distrito Federal, núm. 1689, vol. II (11 de septiembre de 2013), p. 97.
Otro de los aspectos de gran importancia relacionados con la verticalidad en la Ciudad de México es la relación que guarda su naturaleza sísmica con la altura de los edificios. Después del sismo de 1985, los estudios de los edificios desplomados revelaron que las causas no dependieron tanto de la edad de la construcción y el tipo de estructura, sino de la altura, debido a un fenómeno natural conocido como "resonancia", que amplifica el movimiento sísmico debido a la coincidencia de la frecuencia de vibración del terreno con la del edificio. ${ }^{14}$

Este último aspecto da pie para reflexionar en que, a pesar de las bondades de la compactación de la ciudad, no es una receta para cualquier ciudad. Al estar en juego aspectos relacionados con la sociedad, el medio ambiente y la seguridad, la decisión de promover una ciudad vertical va más allá de una función económica o funcional. Una morfología de ciudad que no solo garantice la seguridad, sino también el contacto entre sus habitantes, el uso y presencia de satisfactores urbanos y espacios de convivencia, será la que propicie una verdadera densidad. ${ }^{15}$

\footnotetext{
${ }^{14}$ Roberto Meli, "El sismo de 1985 en México", en José Lugo Hubp y Moshe Inbar (comps.), Desastres naturales en América Latina, 2002, México, FCE, p. 134.

${ }^{15}$ Los resultados aquí presentados son un subproducto del proyecto SIP-IPN 20144135.
} 
Se prohíbe su reproducción total o parcial por cualquier medio, incluido electrónico, sin permiso previo y por escrito de los editores. 